\title{
Full On-chip Nanoliter Immunoassay by Geometrical Magnetic Trapping of Nanoparticle Chains
}

\section{*** Supplementary information ***}

Frédéric Lacharme ${ }^{\mathrm{a}}$, Caroline Vandevyver ${ }^{\mathrm{b}}$, and Martin A. M. Gijs ${ }^{\mathrm{a}}$

${ }^{a}$ Laboratory of Microsystems, Ecole Polytechnique Fédérale de Lausanne (EPFL), CH-1015 Lausanne, Switzerland

${ }^{\mathrm{b}}$ Research commission EPFL-SNF, Ecole Polytechnique Fédérale de Lausanne (EPFL), CH-1015 Lausanne, Switzerland 


\section{Movie S1:}

Movie S1 shows how the chains are retained to the left or the right side of the geometrical traps, depending on the direction of the flow that is visualized by incorporating $1 \mu \mathrm{m}$ fluorescent polystyrene microbeads in a phosphate buffer saline (PBS) solution.

\section{Movie S2:}

Movie S2 shows the fluorescent intensity increase on the nanoparticle chains, when flowing fluorescent detection Ab solution through the microchannel. This fluorescence corresponds to the last step of the immuno-complex formation.

\section{Movie S3:}

Movie S3 shows the fluorescent intensity change, when flowing washing buffer through the microchannel. Aspecifically bound and uncoupled detection antibodies are removed from the microchannel, no surface adsorption is observed. The nanoparticle chains still show a fluorescent signal due to the immunocomplex formation. 


\section{Experimental section}

\section{Purified mAb production}

The purified 5C8 mAb (ATCC HB-10916, directed against the T-B activating molecule T-BAM, ligand for CD40) were prepared by conventional hybridoma technology. Briefly, exhausted serum-free media (Gibco Cell Culture, obtained from Invitrogen, Basel, Switzerland) from static cultures of the mouse hybridoma cell line 5C8 were clarified by centrifugation (3000 g, $20 \mathrm{~min})$ and ultrafiltration $(0.45 \mu \mathrm{m})$, before being purified by protein A affinity chromatography, according to the manufacturer's instruction. The purified mAb $5 \mathrm{C} 8$ were stored at $4{ }^{\circ} \mathrm{C}$ in phosphate buffer at a concentration of $39 \mu \mathrm{g} \cdot \mathrm{mL}^{-1}$.

Non-purified mAb production

Non-purified 5D10 mAb (directed against the human breast cancer cell line MCF-7) was the supernatant of a cell culture. The latter was prepared by conventional hybridoma technology in a conditioned cell culture medium (RPMI 1640, supplemented with $2 \%$ fetal calf serum (FCS), 2 mM L-glutamine, 1 mM sodium pyruvate, 1 \% non-essential amino-acids, 1 \% 4-(2-hydroxyethyl)- monosodium salt (HEPES) (Gibco Cell Culture, Invitrogen, Basel, Switzerland)). The solution was filtered on a $0.45 \mu \mathrm{m}$ pore size membrane before being used in the microchip. 


\section{Figure S1}

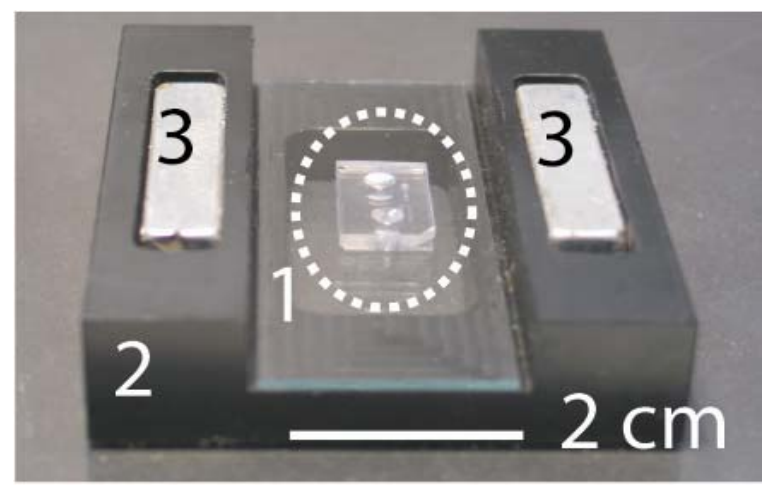

Figure S1: Picture of the microfluidic chip in its socket. The chip consists of a $8 \mu \mathrm{m}$ deep plasma-etched channel in a Pyrex substrate that is sealed with a $2.5 \mathrm{~mm}$ thick Polydimethylsiloxane (PDMS) cover (1). The latter is pinched with 2 access holes and placed on the chip socket (2) that contains 2 permanent magnets (3) generating a homogeneous magnetic field.

\section{Figure S2}

a

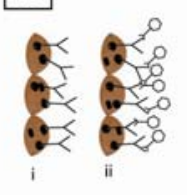

- streptavidin

L Biotinylated capture Ab

Fluorescent rabbit detection $A b$ b

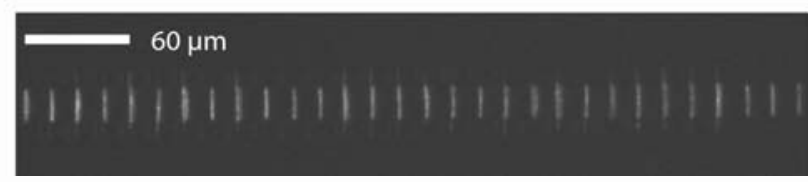

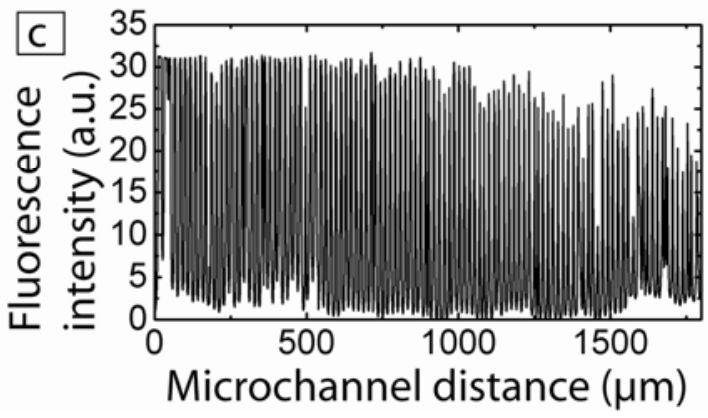

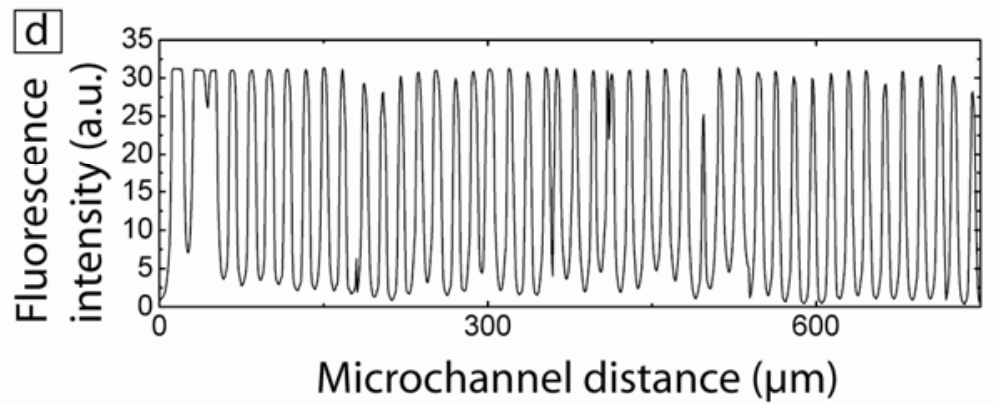

Figure S2: (a) Schematic diagram of the fluorescent direct (not sandwich-type) immunoassay principle. Starting from streptavidin-coated magnetic nanoparticles, biotinylated capture $\mathrm{Ab}$ is linked to the magnetic nanoparticles. Then, fluorescent rabbit detection Ab is flown through the microchannel and bound with the immobilized biotinylated capture Ab. (b) Fluorescent microscopic image of 30 consecutive self-assembled magnetic nanoparticle chains after the realization of the immunoreaction between the biotinylated capture $\mathrm{Ab}$ and the fluorescent rabbit detection $\mathrm{Ab}$. (c) Fluorescent intensity in the microchannel, due to the binding of biotinylated capture Ab and fluorescent rabbit detection Ab on the magnetic nanoparticles. Each peak corresponds to a single fluorescent magnetic chain. (d) Detail of Fig. S2c, showing the saturated fluorescent intensity on the first 50 magnetic chains in the microchannel. 


\section{Magnetic energy calculation}

According to the datasheet of the superparamagnetic streptavidin-coated nanoparticles supplier, Ademtech, the magnetic nanoparticle material has a saturation magnetization $M_{\mathrm{sat}}=8 \times 10^{4} \mathrm{~A} \cdot \mathrm{m}^{-1}$. The magnetic saturation moment of a nanoparticle is $\mathrm{m}_{\text {sat }}=4 / 3 \times \pi \times R^{3} \times M_{\text {sat }}=5.23 \times 10^{-15}$ A.m ${ }^{2}$, with $R$ the nanoparticle radius.

We measured with a miniaturized Hall probe a magnetic induction of $120 \mathrm{mT}$ originating from the two permanent magnets at the center of the chip holder, where the microchannel is inserted during the experiments. This magnetic induction is large enough to saturate the magnetic moment of the nanoparticles. By action of the magnetic field, the saturated magnetic nanoparticles self-assemble in the enlarged cross-sections of the microchannel. The total dipolar interaction energy for a pearl-like chain of $n$ nanoparticles can be calculated using the following relation:

$$
E_{\text {chain }}=\sum_{i=0}^{n} \sum_{j>i}^{n} \frac{1}{4 \pi \mu_{0}} \frac{1}{r_{i j}^{3}}\left(\mathbf{m}_{\mathbf{i}} \cdot \mathbf{m}_{\mathbf{j}}-\frac{3}{r^{2} i j}\left(\mathbf{r}_{\mathbf{i}} \cdot \mathbf{m}_{\mathbf{i}}\right)\left(\mathbf{r}_{\mathbf{j}} \cdot \mathbf{m}_{\mathbf{j}}\right)\right)
$$

where $\mu_{0}$ is the magnetic permeability of vacuum, $\mathbf{m}_{\mathbf{k}}$ and $\mathbf{r}_{\mathbf{k}}$ the magnetic moment and coordinate vector of the nanoparticle $k$, respectively, and $r_{i j}$ is the distance between the nanoparticles with indices $i$ and $j$.

A Mathematica ${ }^{\circledR}$ program was used to calculate this energy, whereby all moments are assumed to be oriented in the direction of the magnetic field, with the modulus of $\mathbf{m}_{\mathbf{k}}=\mathrm{m}_{\mathrm{sat}}=5.23 \times 10^{-15}$ A.m $\mathrm{m}^{2}$. For a chain constituted of 80 pearl-like aligned nanoparticles, we find that $E_{\text {chain }}=-4.3 \times 10^{-15} \mathrm{~J}$.

A liquid flowing through the microchannel will exert a drag force that will displace the 40 nanoparticles of the central part of the magnetic chain over a distance $X$ and eventually breaks up the chain in three parts. With $X$, the magnetic chain energy $E_{\text {chain }}$ increase, leading to a magnetic force retaining the central part of the chain.

The magnetic force energy acting on the central part of the chain is defined as:

$$
F_{\text {mag }}=-\frac{\partial E_{\text {chain }}}{\partial X}
$$

To determine the critical fluid velocity for chain rupture, the maximal magnetic dipolar force of a chain $F_{\operatorname{mag}(\max )}$ needs to be equalized to the drag force.

\section{Drag force calculation}

The viscous drag force acting on the central part of a 80-nanoparticle magnetic chain can be expressed as:

$$
F_{\text {drag }}=N \times 6 \times \pi \times \eta \times R \times v
$$

where $N$ is the number of nanoparticles in the center part of the chain (40 for a 80-nanoparticle pearl-like chain), $\eta$ the liquid viscosity and $v$ the velocity of the liquid with respect to the magnetic nanoparticles. When the drag force is superior to the maximal magnetic force $\left(F_{\text {drag }}>F_{\text {mag }}\right)$, the central part of the magnetic chain is no longer retained and the chain break in 3 parts. 